\title{
Đánh giá phẩm chất mạng chuyển tiếp song công hai chiều trên cùng băng tần dưới tác động của phần cứng không lý tưởng
}

\author{
Nguyễn Bá Cao, Trần Xuân Nam \\ Trường Đại học Kỹ thuật Lê Quý Đôn \\ Tác giả liên hệ: Nguyễn Bá Cao, bacao.sqtt@gmail.com \\ Ngày nhận: 21/04/2018, ngày sửa chữa: 02/08/2018, ngày duyệt đăng: 02/08/2018 \\ Xem sớm trực tuyến: 08/11/2018, định danh DOI: 10.32913/rd-ict.vol1.no39.728 \\ Biên tập lĩnh vực điều phối phản biện và quyết định nhận đăng: TS. Trương Trung Kiên
}

Tóm tắt: Bài báo này phân tích phẩm chất mạng chuyển tiếp song công hai chiều trên cùng băng tần với phần cứng không lý tưởng, trong đó nút chuyển tiếp sử dụng giao thức khuếch đại-và-chuyển tiếp. Xác suất dừng, xác suất lỗi ký hiệu được tìm ra để đánh giá phẩm chất hệ thống. Trên cơ sở đó, để nâng cao phẩm chất hệ thống, chúng tôi tìm ra biểu thức phân bổ công suất tối ưu cho hai nút đầu cuối và nút chuyển tiếp. Kết quả phân tích đã làm rõ mức độ ảnh hưởng của lỗi phần cứng và nhiễu tự giao thoa đến phẩm chất hệ thống khi so sánh với hệ thống lý tưởng và hệ thống bán song công truyền thống. Bên cạnh đó, mô phỏng Monte Carlo được thực hiện để minh chứng cho sự đúng đắn của kết quả phân tích.

Từ khóa: Truyền thông song công trên cùng băng tần, triệt nhiễu tự giao thoa, khuếch đại và chuyển tiếp, xác suất dừng, phần cứng không lý tưởng.

Title: Performance Evaluation of In-Band Full-Duplex Two-Way Relay Networks with Hardware Impairments

Abstract: In this paper, we analyse the performance of in-band full-duplex two-way relay networks using the amplify-and-forward strategy under the impact of hardware impairments. Outage probability and symbol error probability are derived to evaluate the system performance. Based on these values, we derive the optimal power allocation expression for terminal nodes and relay node. Numerical results provide an insight into the impact of hardware impairments and the residual selfinterference on the system performance in comparison with the ideal hardware and the half-duplex systems. Simulation results are used to verify our analyses.

Keywords: In-band full-duplex, self-interference cancellation, amplify-and-forward, outage probability, hardware impairments.

\section{I. ĐẶT VẤN ĐỀ}

Trong giai đoạn cách mạng công nghiệp 4.0 hiện nay, xu thế phát triển về trí tuệ nhân tạo (AI: Machine intelligence), Internet kết nối vạn vật (IoT: Internet of things) và dữ liệu lớn (Big Data) đòi hỏi các thiết bị vô tuyến cần được nâng cấp cả phần cứng lẫn phần mềm để đáp ứng các yêu cầu đặt ra. Nhu cầu dữ liệu lớn trong khi phổ tần hạn chế đòi hỏi các nhà nghiên cứu và thiết kế mạng vô tuyến tìm cách nâng cao hiệu quả sử dụng phổ tần. Trong bối cảnh đó, truyền thông song công trên cùng băng tần (IBFD: In-band fullduplex) là một trong những công nghệ đang được nghiên cứu mạnh bởi khả năng tăng gấp đôi hiệu quả sử dụng phổ tần [1-3]. Những kết quả nghiên cứu và thử nghiệm về khả năng triệt nhiễu tự giao thoa (SIC: Self-Interference Cancellation) ở [4-8] đã chứng minh tính khả thi của truyền thông IBFD. Những nghiên cứu này đã cho thấy nhiễu có thể được loại trừ lên đến $110 \mathrm{~dB}$ thông qua kết hợp các biện pháp SIC, bao gồm khử nhiễu miền ăng-ten, miền số và miền tương tự. Tuy nhiên, do triệt nhiễu không hoàn hảo, phần nhiễu dư còn lại (RSI: Residual self-interference) sẽ làm giảm phẩm chất hệ thống.

Trên cơ sở đó, nhiều nghiên cứu đã thực hiện đánh giá phẩm chất hệ thống FD dưới sự ảnh hưởng của RSI khi so sánh với hệ thống bán song công truyền thống (HD: Halfduplex) nhằm đưa ra phương án lựa chọn sử dụng hệ thống FD hoặc HD trong các điều kiện cụ thể [9-11]. Những công trình này đã cho thấy rằng, khi nhiễu dư FD nhỏ, hệ thống FD cho dung lượng ergodic tốt hơn hệ thống HD với cùng phẩm chất xác suất dừng ( $\mathrm{OP}$ : Outage probability). Ngược lại, khi nhiễu dư FD lớn, sử dụng mô hình HD để nâng cao phẩm chất hệ thống. Đồng thời, sử dụng công 
suất phát thích ứng cho hoạt động HD/FD sẽ giảm được năng lượng tiêu thụ, nâng cao hiệu quả sử dụng phổ tần tức thì.

Trong các nghiên cứu về hệ thống $\mathrm{FD}$, mạng chuyển tiếp (relay network) FD đang được chú trọng bởi khả năng tăng cường độ tin cậy, nâng cao chất lượng, khắc phục hiện tượng che khuất, suy hao truyền dẫn $[1,2]$. Sự kết hợp giữa mạng chuyển tiếp hai chiều và công nghệ FD cho phép nâng cao tốc độ truyền dẫn và hiệu quả sử dụng phổ tần so với hệ thống chuyển tiếp một chiều hoặc hai chiều HD truyền thống. Nhiều nghiên cứu gần đây đã tập trung vào việc đánh giá và nâng cao phẩm chất mạng chuyển tiếp song công hai chiều trong trường hợp triệt nhiễu tự giao thoa không hoàn hảo. Trong các bài báo [1, 2, 12-18], các tác giả đã khảo sát phẩm chất mạng chuyển tiếp FD hai chiều sử dụng giao thức khuếch đại và chuyển tiếp ( $\mathrm{AF}$ : Amplify and forward). Biểu thức $\mathrm{OP}$ trong các điều kiện khác nhau đã được đưa ra và khảo sát ở $[1,13,16,18]$. Các tác giả đã chứng minh được rằng, phẩm chất $\mathrm{OP}$ đạt đến bão hòa khi tỉ số tín hiệu trên nhiễu cộng với tạp âm (SINR: Signal to interferecen plus noise ratio) đủ lớn. Bởi khi SINR đủ lớn, kéo theo tự can nhiễu tại nút chuyển tiếp và hai nút đầu cuối lớn hơn, làm cho OP đạt đến mức sàn lỗi (error floor). Đồng thời, các biện pháp như lựa chọn cặp ăng-ten thu phát, ước lượng kênh nhiễu tự giao thao, và phân bổ công suất tối ưu làm giảm đáng kể ảnh hưởng của RSI và nâng cao phẩm chất hệ thống [12, 14, 15]. Bên cạnh chuyển tiếp $\mathrm{AF}$, mạng giải mã và chuyển tiếp (DF: Decode and forward) hai chiều FD đã và đang được nghiên cứu rộng rãi [19-23]. Các tác giả đã khảo sát OP trong điều kiện CSI hoàn hảo và không hoàn hảo, và khảo sát sự ảnh hưởng của RSI đến phẩm chất hệ thống. Kết quả cho thấy, ảnh hưởng của RSI làm cho phẩm chất hệ thống đạt đến bão hòa ngay cả khi CSI hoàn hảo. Bên cạnh đó, việc phân bổ công suất tối ưu cho nút chuyển tiếp giúp nâng cao đáng kể phẩm chất hệ thống.

Khảo sát trên cho thấy, hầu hết các nghiên cứu về hệ thống FD hai chiều tập trung vào việc phân tích và xem xét ảnh hưởng của các điều kiện về kênh truyền và nhiễu dư đến phẩm chất hệ thống. Tuy nhiên, để hệ thống vô tuyến hoạt động được, ngoài những giải pháp về phần mềm thì phần cứng hệ thống là đòi hỏi bắt buộc. Vì vậy, ngoài các biện pháp kỹ thuật để nâng cao phẩm chất hệ thống, cần xem xét lựa chọn phần cứng phù hợp. Trong thực tế, khi thiết bị vô tuyến hoạt động, các lỗi của phần cứng không hoàn hảo (HI: Hardware impairments) sẽ tạo ra nguồn nhiễu, làm méo tín hiệu cần truyền/nhận. Các kỹ thuật bù trừ được áp dụng để loại bỏ lỗi phần cứng. Dẫu vậy, lỗi phần cứng vẫn sót lại trong các hệ thống vô tuyến và làm giảm phẩm chất hệ thống. Ảnh hưởng của lỗi phần cứng đã được xem xét trong các nghiên cứu $[24,25]$ và một số tài liệu khác.
Kết quả cho thấy rằng, khi lỗi phần cứng tồn tại trong hệ thống, phẩm chất hệ thống sẽ giảm đáng kể, nhất là đối với các hệ thống truyền dẫn tốc độ cao. Đặc biệt là trong mạng chuyển tiếp FD, lỗi phần cứng kết hợp với RSI sẽ làm phẩm chất hệ thống giảm nhanh và sớm đạt đến bão hòa. Điều này đã được một số nhóm nghiên cứu khảo sát như ở [26] và [27]. Trong những nghiên cứu này, mạng chuyển tiếp FD-AF với HI tại nút chuyển tiếp FD đã được phân tích. Trong đó, [26] sử dụng nhiều ăng-ten tại nút chuyển tiếp để nâng cao tốc độ truyền dẫn hệ thống, còn [27] sử dụng nhiều cặp ăng-ten (multipair massive MIMO) để nâng cao hiệu quả sử dụng phổ tần và năng lượng. Kết quả nghiên cứu đã chỉ rõ ảnh hưởng lớn của HI và RSI tại nút chuyển tiếp đến phẩm chất hệ thống, nhất là đối với những thiết bị giá thành thấp.

Xuất phát từ những thảo luận trên, để đánh giá phẩm chất hệ thống sát với điều kiện thực tế, trong bài báo này, chúng tôi khảo sát chất lượng hệ thống chuyển tiếp FD hai chiều $\mathrm{AF}$ dưới tác động của $\mathrm{HI}$ và RSI ở cả nút chuyển tiếp và hai nút đầu cuối. Xác suất dừng, xác suất lỗi ký hiệu và phân bổ công suất tối ưu cho hệ thống được sử dụng để đánh giá, và để nâng cao phẩm chất hệ thống.

Bài báo có ba đóng góp chính sau. Trước hết, chúng tôi đã xây dựng mô hình mạng chuyển tiếp FD hai chiều sử dụng giao thức $\mathrm{AF}$ với phần cứng không lý tưởng để khảo sát. Tiếp theo, trên cơ sở mô hình hệ thống và tín hiệu, chúng tôi đưa ra biểu thức chính xác của tỉ số công suất tín hiệu trên nhiễu cộng tạp âm và méo dạng (SINDR: Signal to interference plus noise and distortion ratio) được đưa ra, làm cơ sở để tìm ra biểu thức chính xác và xấp xỉ của $\mathrm{OP}$, và biểu thức xấp xỉ của xác suất lỗi ký hiệu (SEP: Symbol error probability). Từ đó, phẩm chất hệ thống được đánh giá toàn diện, cho phép xác định được ảnh hưởng của lỗi phần cứng và RSI đến phẩm chất hệ thống khi so sánh với hệ thống lý tưởng và hệ thống HD. Cuối cùng, để nâng cao phẩm chất hệ thống, chúng tôi đề xuất phân bổ công suất tối ưu cho hai nút đầu cuối và nút chuyển tiếp, nhằm làm giảm tác động của lỗi phần cứng và RSI. Kết quả cho thấy, phương án đề xuất làm tăng đáng kể phẩm chất hệ thống.

Phần còn lại của bài báo được tổ chức như sau. Mục II là mô hình hệ thống và tín hiệu, Mục III tập trung vào việc phân tích phẩm chất hệ thống, Mục IV đề xuất phân bổ công suất tối ưu cho hệ thống, Mục $\mathrm{V}$ trình bày kết quả tính toán số và thảo luận, và cuối cùng, Mục VI rút ra các kết luận của bài báo.

\section{MÔ HÌNH HÊ̂ THỐNG}

Xét một hệ thống liên lạc điểm - điểm với phần cứng lý tưởng (gọi tắt là hệ thống lý tưởng), trong đó mỗi đầu 
cuối có một ăng-ten dùng cho thu và phát tín hiệu ở chế độ HD, tín hiệu thu có dạng sau:

$$
y=h s+z,
$$

trong đó $h$ là hệ số pha đinh của kênh truyền từ phía phát đến phía thu, $s$ là tín hiệu cần truyền đi, $z$ là nhiễu trắng cộng tính (AWGN: Additive white Gaussian noise) tại đầu vào máy thu. Trong trường hợp phần cứng không lý tưởng (gọi tắt là hệ thống không lý tưởng), phía máy phát và máy thu sẽ tạo ra các nguồn nhiễu lần lượt là $\eta^{\text {tx }}$ và $\eta^{\text {rx }}$, với $\eta^{\mathrm{tx}} \sim C \mathcal{N}\left(0, k_{\mathrm{tx}}^{2} P\right), \eta^{\mathrm{rx}} \sim C \mathcal{N}\left(0, k_{\mathrm{rx}}^{2}|h|^{2} P\right)$ [24]. Trong đó, $k_{\mathrm{tx}}$ và $k_{\mathrm{rx}}$ lần lượt là các tham số đặc trưng cho lỗi phần cứng ở máy phát và máy thu; $P$ là công suất tín hiệu phát. Từ đó, tín hiệu thu được có dạng sau:

$$
y=h\left(s+\eta^{\mathrm{tx}}\right)+\eta^{\mathrm{rx}}+z=h s+h \eta^{\mathrm{tx}}+\eta^{\mathrm{rx}}+z .
$$

Từ (2), công suất trung bình của lỗi phần cứng tổng hợp do cả phía phát và phía thu gây nên được biểu diễn bằng $\mathbb{E}_{\eta^{\mathrm{tx}}, \eta^{\mathrm{rx}}}\left\{\left|h \eta^{\mathrm{tx}}+\eta^{\mathrm{rx}}\right|^{2}\right\}=P|h|^{2}\left(k_{\mathrm{tx}}^{2}+k_{\mathrm{rx}}^{2}\right)=P|h|^{2} k^{2}$, với $k^{2}=$ $k_{\mathrm{tx}}^{2}+k_{\mathrm{rx}}^{2}$ là méo dạng tổng hợp, đặc trưng cho mức độ ảnh hưởng của lỗi phần cứng tổng hợp ở cả máy phát và máy thu. Từ đó, (2) được đơn giản hóa thành

$$
y=h(s+\eta)+z
$$

với $\eta \sim C \mathcal{N}\left(0, k^{2} P\right)$. Trên cơ sở mô hình cho hệ thống điểm - điểm này, chúng tôi xây dựng mô hình hệ thống chuyển tiếp song công hai chiều với phần cứng không lý tưởng như chỉ ra trên Hình 1. Trong mô hình này, để biểu diễn RSI ở chế độ FD, chúng tôi sử dụng hai ký hiệu ăngten biểu diễn cho hai mạch thu và phát. Hệ thống thực tế có thể sử dụng một ăng ten chung cho cả thu và phát. Dữ liệu được truyền từ $S_{1}$ tới $S_{2}$ và ngược lại thông qua trạm chuyển tiếp $\mathrm{R}$. Trong đó, $\mathrm{R}$ sử dụng giao thức khuếch đại và chuyển tiếp.

Tại thời điểm $t$, tín hiệu thu của nút chuyển tiếp có dạng

$$
y_{\mathrm{R}}(t)=h_{1 \mathrm{R}} \widetilde{s}_{1}(t)+h_{2 \mathrm{R}} \widetilde{s}_{2}(t)+\widetilde{h}_{\mathrm{RR}} \widetilde{s}_{\mathrm{R}}(t)+z_{\mathrm{R}}(t),
$$

ở đây $\widetilde{s}_{1}(t) \triangleq s_{1}(t)+\eta_{1}(t), \widetilde{s}_{2}(t) \triangleq s_{2}(t)+\eta_{2}(t), \widetilde{s}_{\mathrm{R}}(t) \triangleq$ $s_{\mathrm{R}}(t)+\eta_{\mathrm{R}}(t)$, với $\widetilde{s}_{1}, \widetilde{s}_{2}$ và $\widetilde{s}_{\mathrm{R}}$ lần lượt là các tín hiệu thực tế phát đi tại $\mathrm{S}_{1}, \mathrm{~S}_{2}$ và $\mathrm{R} ; s_{1}, s_{2}$ và $s_{\mathrm{R}}$ lần lượt là các tín hiệu

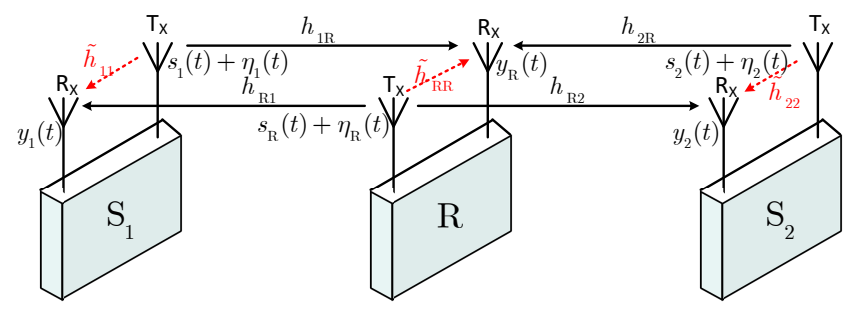

Hình 1. Hệ thống chuyển tiếp song công hai chiều với phần cứng không lý tưởng. mong muốn phát đi tại $\mathrm{S}_{1}, \mathrm{~S}_{2}$ và $\mathrm{R} ; \eta_{1}, \eta_{2}$ và $\eta_{\mathrm{R}}$ lần lượt là các lỗi phần cứng tổng hợp tại $\mathrm{S}_{1}, \mathrm{~S}_{2}$ và $\mathrm{R}$ thỏa mãn $\eta_{1} \sim$ $C \mathcal{N}\left(0, k_{1}^{2} P_{1}\right), \eta_{2} \sim C \mathcal{N}\left(0, k_{2}^{2} P_{2}\right)$ và $\eta_{\mathrm{R}} \sim C \mathcal{N}\left(0, k_{\mathrm{R}}^{2} P_{\mathrm{R}}\right)$, với $k_{1}, k_{2}$ và $k_{\mathrm{R}}$ lần lượt là hệ số méo dạng tổng hợp tại $\mathrm{S}_{1}, \mathrm{~S}_{2}$ và $\mathrm{R} ; P_{1}, P_{2}$ và $P_{\mathrm{R}}$ lần lượt là công suất phát trung bình tại $\mathrm{S}_{1}, \mathrm{~S}_{2}$ và $\mathrm{R} ; h_{1 \mathrm{R}}, h_{2 \mathrm{R}}$ và $\widetilde{h}_{\mathrm{RR}}$ tương ứng là hệ số pha-đinh của kênh truyền từ $S_{1}$ tới $R$, từ $S_{2}$ tới $R$ và từ kênh ảnh hưởng mạch phát tới mạch thu của $\mathrm{R} ; z_{\mathrm{R}}(t)$ là nhiễu trắng cộng tính (AWGN) tại đầu vào $\mathrm{R}$, với $z_{\mathrm{R}} \sim C \mathcal{N}\left(0, N_{\mathrm{R}}\right)$.

Giả sử rằng, thời gian xử lý tín hiệu tại $\mathrm{R}$ bằng một chu kỳ ký hiệu, tức là thành phần tín hiệu phát trên nút chuyển tiếp $\widetilde{s}_{\mathrm{R}}(t)$ được thực hiện sau khi khuếch đại tín hiệu thu ở thời điểm trước đó $y_{\mathrm{R}}(t-1)$, kết hợp với méo do lỗi phần cứng của máy thu phát. Do đó ta có

$$
\tilde{s}_{\mathrm{R}}(t)=G y_{\mathrm{R}}(t-1)+\eta_{\mathrm{R}}(t-1) .
$$

Khi thông tin trạng thái kênh truyền hoàn hảo, hệ số khuếch đại $G$ được định nghĩa theo biểu thức sau:

$$
G \triangleq \sqrt{\frac{P_{\mathrm{R}}}{\rho_{1} P_{1}\left(1+k_{1}^{2}\right)+\rho_{2} P_{2}\left(1+k_{2}^{2}\right)+I_{\mathrm{R}}+N_{\mathrm{R}}}},
$$

trong đó, $\rho_{1}=\left|h_{1 R}\right|^{2}$ và $\rho_{2}=\left|h_{2 R}\right|^{2}$ lần lượt là các độ lợi kênh truyền từ $\mathrm{S}_{1}$ đển $\mathrm{R}$ và từ $\mathrm{S}_{2}$ đến $\mathrm{R} ; I_{\mathrm{R}}=\widetilde{\Omega}_{\mathrm{R}} P_{\mathrm{R}}$ là nhiễu lặp vòng còn sót lại tại $\mathrm{R}$ sau khi áp dụng tất cả các kỹ thuật triệt nhiễu tự giao thoa, với $\widetilde{\Omega}_{R}$ biểu thị khả năng triệt nhiễu tại R.

Sau khi khuếch đại, $\mathrm{R}$ chuyển tiếp tín hiệu thu được tới $S_{1}$ và $S_{2}$. Tín hiệu thu tại $S_{2}$ có dạng sau:

$$
y_{2}(t)=h_{\mathrm{R} 2} \widetilde{s}_{\mathrm{R}}(t)+\widetilde{h}_{22} \widetilde{s}_{2}(t)+z_{2}(t),
$$

trong đó, $h_{\mathrm{R} 2}$ và $\widetilde{h}_{22}$ lần lượt là hệ số pha đinh của kênh truyền từ $R$ tới $S_{2}$ và từ mạch phát đến mạch thu của $S_{2}$; $z_{2}$ là nhiễu AWGN tại $S_{2}$. Thế (5) vào (7) ta được

$$
y_{2}(t)=h_{\mathrm{R} 2}\left[G y_{\mathrm{R}}(t-1)+\eta_{\mathrm{R}}(t-1)\right]+\widetilde{h}_{22} \widetilde{s}_{2}(t)+z_{2}(t) .
$$

Sử dụng (4), biểu thức (8) trở thành

$$
\begin{aligned}
y_{2}(t) & =h_{\mathrm{R} 2} G\left[h_{1 \mathrm{R}}\left(s_{1}(t-1)+\eta_{1}(t-1)\right)+h_{2 \mathrm{R}} s_{2}(t-1)\right] \\
& +h_{\mathrm{R} 2} G\left[h_{2 \mathrm{R}} \eta_{2}(t-1)+\widetilde{h}_{\mathrm{RR}} \widetilde{s}_{\mathrm{R}}(t-1)+z_{\mathrm{R}}(t-1)\right] \\
& +h_{\mathrm{R} 2} \eta_{\mathrm{R}}(t-1)+\widetilde{h}_{22} \widetilde{s}_{2}(t)+z_{2}(t) .
\end{aligned}
$$

Sử dụng kỹ thuật mã hóa mạng [2], ta có thể loại bỏ được thành phần $s_{2}(t-1)$ ra khỏi $y_{2}(t)$, đồng thời có $\left|h_{\mathrm{R} 2}\right|^{2}=$ $\left|h_{2 \mathrm{R}}\right|^{2}$ và $\left|h_{\mathrm{R} 1}\right|^{2}=\left|h_{1 \mathrm{R}}\right|^{2}$. Do đó, ta xác định được SINDR tại $\mathrm{S}_{2}$ theo biểu thức

$$
\gamma_{2}=\frac{\left|h_{1 \mathrm{R}}\right|^{2}\left|h_{\mathrm{R} 2}\right|^{2} P_{1}}{\left|h_{1 \mathrm{R}}\right|^{2}\left|h_{\mathrm{R} 2}\right|^{2} P_{1} d+\left|h_{\mathrm{R} 2}\right|^{2}\left|h_{2 \mathrm{R}}\right|^{2} P_{2} d^{\prime}+\phi_{1}},
$$

hay

$$
\gamma_{2}=\frac{\rho_{1} \rho_{2} P_{1} P_{\mathrm{R}}}{\rho_{1} P_{1}\left[\rho_{2} P_{\mathrm{R}} d+t_{2} d_{1}\right]+\rho_{2}^{2} P_{2} P_{\mathrm{R}} d^{\prime}+\phi_{2}} .
$$


Tương tự ta có SINDR tại $S_{1}$ như sau:

$$
\gamma_{1}=\frac{\rho_{1} \rho_{2} P_{2} P_{\mathrm{R}}}{\rho_{2} P_{2}\left[\rho_{1} P_{\mathrm{R}} d^{\prime}+t_{1} d_{2}\right]+\rho_{1}^{2} P_{1} P_{\mathrm{R}} d+\phi_{3}},
$$

trong đó: $\phi_{1}=\left|h_{\mathrm{R} 2}\right|^{2} t_{\mathrm{R}} d_{\mathrm{R}}+t_{2} / G^{2}, \phi_{2}=\rho_{2} P_{\mathrm{R}} t_{\mathrm{R}} d_{\mathrm{R}}+$ $\rho_{2} P_{2} t_{2} d_{2}+t_{2} t_{\mathrm{R}}, \phi_{3}=\rho_{1} P_{\mathrm{R}} t_{\mathrm{R}} d_{\mathrm{R}}+\rho_{1} P_{1} t_{1} d_{1}+t_{1} t_{\mathrm{R}}, \rho_{1}=$ $\left|h_{1 \mathrm{R}}\right|^{2}=\left|h_{\mathrm{R} 1}\right|^{2}, \rho_{2}=\left|h_{\mathrm{R} 2}\right|^{2}=\left|h_{2 \mathrm{R}}\right|^{2}, d=k_{1}^{2}+k_{\mathrm{R}}^{2}+k_{1}^{2} k_{\mathrm{R}}^{2}$, $d^{\prime}=k_{2}^{2}+k_{\mathrm{R}}^{2}+k_{2}^{2} k_{\mathrm{R}}^{2}, d_{1}=1+k_{1}^{2}, d_{2}=1+k_{2}^{2}, d_{\mathrm{R}}=1+k_{\mathrm{R}}^{2}$, $I_{1}=\widetilde{\Omega}_{1} P_{1}, I_{2}=\widetilde{\Omega}_{2} P_{2}, I_{\mathrm{R}}=\widetilde{\Omega}_{\mathrm{R}} P_{\mathrm{R}}, t_{1}=I_{1}+N_{1}, t_{2}=I_{2}+N_{2}$, $t_{\mathrm{R}}=I_{\mathrm{R}}+N_{\mathrm{R}}$, với $\widetilde{\Omega}_{1}$ và $\widetilde{\Omega}_{2}$ lần lượt biểu thị khả năng triệt nhiễu tại $S_{1}$ và $S_{2}$.

Chú ý rằng, trong trường hợp $k_{1}=k_{2}=k_{\mathrm{R}}=0$, hệ thống này trở thành hệ thống lý tưởng.

\section{PHÂN TÍCH PHẨM CHẤT HÊ THỐNG}

\section{Xác suất dừng hệ thống}

1) Biểu thức chinh xác xác suất dùng:

Xác suất dừng hệ thống (OP: Outage probability) được định nghĩa là xác suất mà tỉ số SINDR nhỏ hơn mức ngưỡng yêu cầu tối thiểu hệ thống đặt ra, tức là

$$
P_{\text {out }}=\operatorname{Pr}\{\gamma<x\},
$$

trong đó $\gamma$ là tỉ số SINDR và $x$ là mức ngưỡng xem xét, với $x=2^{\mathcal{R}}-1$, ở đây $\mathcal{R}$ là tốc độ yêu cầu tối thiểu của hệ thống có đơn vị là bit/s/Hz. Từ đó xác suất dừng tại $\mathrm{S}_{2}$ được xác định như sau:

$$
P_{\text {out }}^{\mathrm{S}_{2}}=\operatorname{Pr}\left\{\gamma_{2}<x\right\}=1-\int_{0}^{\infty}\left[1-F_{\rho_{1}}(X)\right] f_{y}(y) d y,
$$

với $X$ được xác định bằng

$$
\begin{aligned}
X & =\frac{2 x^{2} P_{2} t_{2} d_{1} d^{\prime}}{P_{1} P_{\mathrm{R}}(1-x d)^{2}}+\frac{x\left(P_{\mathrm{R}} t_{\mathrm{R}} d_{\mathrm{R}}+P_{2} t_{2} d_{2}\right)}{P_{1} P_{\mathrm{R}}(1-x d)} \\
& +\frac{x^{2} t_{2} d_{1}\left(P_{\mathrm{R}} t_{\mathrm{R}} d_{\mathrm{R}}+P_{2} t_{2} d_{2}\right)}{y P_{1} P_{\mathrm{R}}^{2}(1-x d)^{2}}+\frac{x t_{2} t_{\mathrm{R}}}{y P_{1} P_{\mathrm{R}}(1-x d)} \\
& +\frac{x y P_{2} d^{\prime}}{P_{1}(1-x d)}+\frac{x^{3} P_{2} t_{2}^{2} d_{1}^{2} d^{\prime}}{y P_{1} P_{\mathrm{R}}^{2}(1-x d)^{3}} .
\end{aligned}
$$

Trong biểu thức (14), $F_{\rho_{1}}(X)$ và $f_{y}(y)$ lần lượt biểu thị hàm phân phối tích lũy (CDF: Cumulative distribution function) và hàm mật độ xác suất (PDF: Probability density function), với $y=\rho_{2}-\frac{x t_{2} d_{1}}{P_{\mathrm{R}}(1-x d)}$.

Chú ý rằng, khi kênh truyền là kênh pha-đinh Rayleigh, hàm $\mathrm{CDF}$ có dạng:

$$
F_{\rho_{l}}(x)=1-e^{-\frac{x}{\Omega_{l}}}, x \geq 0, l=1 \div 2,
$$

với $\Omega_{l}=\mathbb{E}\left\{\left|h_{l}\right|^{2}\right\}$ là độ lợi trung bình của kênh truyền.
Từ biểu thức (14) và (15), sau một số phép biến đổi, áp dụng công thức (3.324) trong [28] ta xác định được OP như sau:

$$
P_{\text {out }}^{S_{2}}(x)= \begin{cases}1-2 \frac{1-x d}{1+C_{2} x} e^{-\frac{A_{2} x}{1-x d}-\frac{B_{2} x^{2}}{(1-x d)^{2}}} U_{2}(x), & x<1 / d, \\ 1, & x \geq 1 / d .\end{cases}
$$

trong đó, với $\Omega_{1}=\mathbb{E}\left\{\rho_{1}\right\}$ và $\Omega_{2}=\mathbb{E}\left\{\rho_{2}\right\}$, ta có

$$
\begin{aligned}
A_{2} & \triangleq \frac{t_{\mathrm{R}} d_{\mathrm{R}}}{\Omega_{1} P_{1}}+\frac{t_{2}\left(\Omega_{2} P_{2} d_{2}+\Omega_{1} P_{1} d_{1}\right)}{\Omega_{1} P_{1} \Omega_{2} P_{\mathrm{R}}} ; \\
B_{2} & \triangleq \frac{2 P_{2} t_{2} d^{\prime} d_{1}}{\Omega_{1} P_{1} P_{\mathrm{R}}} ; \\
C_{2} & \triangleq \frac{d^{\prime} \Omega_{2} P_{2}-d \Omega_{1} P_{1}}{\Omega_{1} P_{1}} ; \\
U_{2}(x) & \triangleq \sqrt{M_{2}(x)} K_{1}\left(2 \sqrt{M_{2}(x)}\right) \\
M_{2}(x) & \triangleq\left[\frac{D_{2} x^{3}}{(1-x d)^{3}}+\frac{E_{2} x^{2}}{(1-x d)^{2}}+\frac{F_{2} x}{1-x d}\right] \frac{1+C_{2} x}{1-x d} ; \\
D_{2} & \triangleq \frac{P_{2} t_{2}^{2} d_{1}^{2} d^{\prime}}{\Omega_{1} P_{1} \Omega_{2} P_{\mathrm{R}}^{2}} ; \\
E_{2} & \triangleq \frac{t_{2} d_{1}\left(P_{\mathrm{R}} t_{\mathrm{R}} d_{\mathrm{R}}+P_{2} t_{2} d_{2}\right)}{\Omega_{1} P_{1} \Omega_{2} P_{\mathrm{R}}^{2}} ; \\
F_{2} & \triangleq \frac{t_{2} t_{\mathrm{R}}}{\Omega_{1} P_{1} \Omega_{2} P_{\mathrm{R}}} .
\end{aligned}
$$

Bằng phương pháp tương tự, ta xác định được xác suất dừng tại $\mathrm{S}_{1}$ như sau:

$$
P_{\text {out }}^{\mathrm{S}_{1}}(x)= \begin{cases}1-2 \frac{1-x d^{\prime}}{1+C_{1} x} e^{-\frac{A_{1} x}{1-x d^{\prime}}-\frac{B_{1} x^{2}}{\left(1-x d^{\prime}\right)^{2}}} U_{1}(x), & x<1 / d^{\prime}, \\ 1, & x \geq 1 / d^{\prime}\end{cases}
$$

trong đó

$$
\begin{aligned}
A_{1} & \triangleq \frac{t_{\mathrm{R}} d_{\mathrm{R}}}{\Omega_{2} P_{2}}+\frac{t_{1}\left(\Omega_{1} P_{1} d_{1}+\Omega_{2} P_{2} d_{2}\right)}{\Omega_{1} P_{\mathrm{R}} \Omega_{2} P_{2}} ; \\
B_{1} & \triangleq \frac{2 P_{1} t_{1} d d_{2}}{\Omega_{2} P_{2} P_{\mathrm{R}}} ; \\
C_{1} & \triangleq \frac{d \Omega_{1} P_{1}-d^{\prime} \Omega_{2} P_{2}}{\Omega_{2} P_{2}} ; \\
U_{1}(x) & \triangleq \sqrt{M_{1}(x) K_{1}}\left(2 \sqrt{M_{1}(x)}\right) ; \\
M_{1}(x) & \triangleq\left[\frac{D_{1} x^{3}}{\left(1-x d^{\prime}\right)^{3}}+\frac{E_{1} x^{2}}{\left(1-x d^{\prime}\right)^{2}}+\frac{F_{1} x}{1-x d^{\prime}}\right] \frac{1+C_{1} x}{1-x d^{\prime}} ; \\
D_{1} & \triangleq \frac{P_{1} t_{1}^{2} d_{2}^{2} d}{\Omega_{1} P_{2} \Omega_{2} P_{\mathrm{R}}^{2}} ; \\
E_{1} & \triangleq \frac{t_{1} d_{2}\left(P_{\mathrm{R}} t_{\mathrm{R}} d_{\mathrm{R}}+P_{1} t_{1} d_{1}\right)}{\Omega_{1} P_{2} \Omega_{2} P_{\mathrm{R}}^{2}} \\
F_{1} & \triangleq \frac{t_{1} t_{\mathrm{R}}}{\Omega_{1} P_{2} \Omega_{2} P_{\mathrm{R}}} .
\end{aligned}
$$


Trong trường hợp phần cứng hệ thống là lý tưởng, biểu thức (17) được đơn giản hóa thành

$$
P_{\text {out }}^{\text {Sid }}(x)=1-2 e^{-A_{2}^{\mathrm{id}} x} \sqrt{M_{2}^{\mathrm{id}}(x)} K_{1}\left(2 \sqrt{M_{2}^{\mathrm{id}}(x)}\right),
$$

trong đó

$$
\begin{aligned}
A_{2}^{\mathrm{id}} & \triangleq \frac{t_{\mathrm{R}}}{\Omega_{1} P_{1}}+\frac{t_{2}\left(\Omega_{2} P_{2}+\Omega_{1} P_{1}\right)}{\Omega_{1} P_{1} \Omega_{2} P_{\mathrm{R}}} ; \\
B_{2}^{\mathrm{id}} & =C_{2}^{\mathrm{id}}=D_{2}^{\mathrm{id}}=0 ; \\
E_{2}^{\mathrm{id}} & \triangleq \frac{t_{2}\left(P_{\mathrm{R}} t_{\mathrm{R}}+P_{2} t_{2}\right)}{\Omega_{1} P_{1} \Omega_{2} P_{\mathrm{R}}^{2}} ; \\
F_{2}^{\mathrm{id}} & \triangleq \frac{t_{2} t_{\mathrm{R}}}{\Omega_{1} P_{1} \Omega_{2} P_{\mathrm{R}}} ; \\
M_{2}^{\mathrm{id}}(x) & \triangleq E_{2}^{\mathrm{id}} x^{2}+F_{2}^{\mathrm{id}} x .
\end{aligned}
$$

Biểu thức $\mathrm{OP}$ tại $\mathrm{S}_{1}$ có dạng tương tự.

2) Biểu thức xấp xỉ xác suất dùng:

Để thu được biểu thức dạng đơn giản hơn của OP ở (17) và (18), trong phần này, chúng tôi đưa ra biểu thức xấp xỉ cho trường hợp công suất phát lớn. Khi công suất phát đủ lớn, giá trị $M_{1}(x)$ và $M_{2}(x)$ trong biểu thức (17) và (18) rất nhỏ, sử dụng biểu thức xấp xỉ $K_{1}(u) \approx 1 / u$ khi $u \ll 1$, kết hợp với hàm xấp xỉ Taylor $e^{v} \approx 1+v$ khi $v \rightarrow 0$, ta xác định được biểu thức xấp xỉ trong trường hợp $x<1 / d^{\prime}$ hoặc $x<1 / d$ như sau:

$$
\begin{aligned}
& P_{\text {out }}^{\mathrm{S}_{1}^{\mathrm{ap}}}(x) \approx 1-e^{-\left(d^{\prime}+C_{1}\right) x}+A_{1} x e^{-C_{1} x}+B_{1} x^{2} e^{\left(d^{\prime}-C_{1}\right) x}, \\
& P_{\text {out }}^{\mathrm{S}_{2}^{\mathrm{ap}}}(x) \approx 1-e^{-\left(d+C_{2}\right) x}+A_{2} x e^{-C_{2} x}+B_{2} x^{2} e^{\left(d-C_{2}\right) x} .
\end{aligned}
$$

\section{Phẩm chất lỗi ký hiệu}

Theo [2], tỉ lệ lỗi ký hiệu trung bình được xác định thông qua biểu thức:

$$
\mathrm{SEP}=\alpha \mathbb{E}\{Q(\sqrt{\beta \gamma})\}=\frac{\alpha}{\sqrt{2 \pi}} \int_{0}^{\infty} F\left(\frac{t^{2}}{\beta}\right) e^{-\frac{t^{2}}{2}} d t,
$$

trong đó,

$$
Q(x)=\frac{1}{\sqrt{2 \pi}} \int_{x}^{\infty} e^{-t^{2} / 2} d t
$$

giá trị $\gamma$ là SINDR của hệ thống, $\alpha$ và $\beta$ được xác định thông qua dạng điều chế (chẳng hạn, với điều chế BFSK trực giao, $\alpha=\beta=1$, với điều chế BPSK, $\alpha=1, \beta=2$ ), $F(x)$ là $\mathrm{CDF}$ của SINDR và bằng $P_{\mathrm{out}}(x)$ với $P_{\mathrm{out}}(x)$ được xác định từ biểu thức (17) và (18). Đặt $x=t^{2} / \beta$, ta xác định được SEP thông qua biểu thức

$$
\mathrm{SEP}=\frac{\alpha \sqrt{\beta}}{2 \sqrt{2 \pi}} \int_{0}^{\infty} \frac{e^{-\beta x / 2}}{\sqrt{x}} F(x) d x .
$$

Do tính phức tạp của biểu thức OP ở (17) và (18) nên việc đưa ra biểu thức chính xác từ (23) là không thực hiện được. Do đó, sử dụng biểu thức xấp xỉ ở (20) và (21) thay vào biểu thức (23) để tìm biểu thức gần đúng tỉ lệ lỗi ký hiệu. Thông qua phép biến đổi tích phân từng phần, kết hợp với áp dụng biểu thức (3.361.1) và (3.361.2) trong [28], ta xác định được biểu thức gần đúng $\mathrm{SEP}$ tại $\mathrm{S}_{1}$ và $\mathrm{S}_{2}$ tương ứng như sau:

$$
\begin{aligned}
& \operatorname{SEP}_{1} \approx \frac{\alpha \sqrt{\beta}}{2 \sqrt{2 \pi}}\left\{\sqrt{\frac{2 \pi}{\beta}}-\sqrt{\frac{2 \pi}{C_{1}^{\beta}+2 d^{\prime}}} \operatorname{erf}\left(\sqrt{\frac{C_{1}^{\beta}+2 d^{\prime}}{2 d^{\prime}}}\right)\right. \\
&-\frac{2 A_{1} e^{-\frac{C_{1}^{\beta}}{2 d^{\prime}}}}{C_{1}^{\beta} \sqrt{d^{\prime}}}+\frac{A_{1}}{C_{1}^{\beta}} \sqrt{\frac{2 \pi}{C_{1}^{\beta}}} \operatorname{erf}\left(\sqrt{\frac{C_{1}^{\beta}}{2 d^{\prime}}}\right) \\
&+\frac{B_{1}}{\left(C_{1}^{\beta}-2 d^{\prime}\right)^{2}}\left[\frac{-2\left(C_{1}^{\beta}+d^{\prime}\right) e^{-\frac{C_{1}^{\beta}-2 d^{\prime}}{2 d^{\prime}}}}{\sqrt{d^{\prime 3}}}\right. \\
&\left.\left.+3 \sqrt{\frac{2 \pi}{C_{1}^{\beta}-2 d^{\prime}}} \operatorname{erf}\left(\sqrt{\frac{C_{1}^{\beta}-2 d^{\prime}}{2 d^{\prime}}}\right)\right]\right\} \\
& \mathrm{SEP}_{2} \approx \frac{\alpha \sqrt{\beta}}{2 \sqrt{2 \pi}}\left\{\sqrt{\frac{2 \pi}{\beta}}-\sqrt{\frac{2 \pi}{C_{2}^{\beta}+2 d}} \operatorname{erf}\left(\sqrt{\frac{C_{2}^{\beta}+2 d}{2 d}}\right)\right. \\
&-\frac{2 A_{2} e^{-\frac{C_{2}^{\beta}}{2 d}}}{C_{2}^{\beta} \sqrt{d}}+\frac{A_{2}}{C_{2}^{\beta}} \sqrt{\frac{2 \pi}{C_{2}^{\beta}} \operatorname{erf}\left(\sqrt{\frac{C_{2}^{\beta}}{2 d}}\right)} \\
&+\frac{B_{2}}{\left(C_{2}^{\beta}-2 d\right)^{2}}\left[\frac{-2\left(C_{2}^{\beta}+d\right) e^{-\frac{C_{2}^{\beta}-2 d}{2 d}}}{\sqrt{d^{3}}}\right. \\
&\left.\left.\operatorname{linf}\left(\sqrt{\frac{C_{2}^{\beta}-2 d}{2 d}}\right)\right]\right\},
\end{aligned}
$$

trong đó, erf là hàm lỗi (error function) được xác định bởi $\operatorname{erf}(x)=\frac{2}{\sqrt{\pi}} \int_{0}^{x} e^{-t^{2}} d t, C_{1}^{\beta}=\beta+2 C_{1}$ và $C_{2}^{\beta}=\beta+2 C_{2}$.

Trong trường hợp phần cứng là lý tưởng, xác suất lỗi ký hiệu tại $\mathrm{S}_{2}$ có dạng đơn giản là

$$
\mathrm{SEP}_{2}^{\mathrm{id}} \approx \frac{\alpha A_{2}^{\text {id }}}{2 \beta},
$$

và tại $\mathrm{S}_{1}$ có dạng tương tự.

\section{TỐI ƯU HÓA PHÂN BỔ CÔNG SUẤT CHO HÊ THỐNG}

Đối với truyền thông song công trên cùng băng tần, độ lớn của nhiễu tự giao thoa còn sót lại quyết định đến sự tồn tại của hệ thống. Để giảm ảnh hưởng của nhiễu tự giao thoa, nâng cao phẩm chất hệ thống, việc phân bổ công suất phát hợp lý là điều hết sức cần thiết. Vì vậy, cần tính toán để phân bổ công suất phát cho nút chuyển tiếp và hai nút đầu cuối phù hợp. 
Để thuận tiện cho việc tính toán, chúng tôi giả sử rằng $\Omega_{1}=\Omega_{2}, \widetilde{\Omega}_{1}=\widetilde{\Omega}_{2}, N_{1}=N_{2}, k_{1}=k_{2}$ và $P_{1}=P_{2}$. Như vậy, mô hình khảo sát trở thành mô hình đối xứng (symmetric), tức là mô hình có hai nút đầu cuối giống hệt nhau. Từ đó ta có: $d=d^{\prime}, d_{1}=d_{2}, t_{1}=t_{2}, A_{1}=A_{2}, B_{1}=B_{2}$ và $C_{1}=C_{2}=0$. Chúng tôi xem xét tổng công suất phát của hệ thống là $P_{1}+P_{2}+P_{\mathrm{R}}=P$. Đặt $P_{1}=P_{2}=\lambda P$, từ đó $P_{\mathrm{R}}=(1-2 \lambda) P$.

Với những điều kiện trên, (18) được giản lược thành

$$
P_{\text {out }}^{\mathrm{S}_{1}}(x) \approx 1-(1-x d) e^{-\frac{A_{1} x}{1-x d}-\frac{B_{1} x^{2}}{(1-x d)^{2}}}=f(\lambda),
$$

cho trường hợp $x<1 / d$. Lấy đạo hàm theo $\lambda$ ta có

$$
f^{\prime}(\lambda)=x(1-x d) e^{-\frac{A_{1} x}{1-x d}-\frac{B_{1} x^{2}}{(1-x d)^{2}}}\left[\frac{A_{1}^{\prime}}{1-x d}+\frac{B_{1}^{\prime} x}{(1-x d)^{2}}\right],
$$

trong $A_{1}^{\prime}$ và $B_{1}^{\prime}$ lần lượt là đạo hàm của $A_{1}$ và $B_{1}$ theo $\lambda$. Sau một số phép biến đổi toán học, cho $f^{\prime}(\lambda)=0$, ta thu được phương trình sau:

$$
a \lambda^{2}+b \lambda+c=0
$$

trong đó:

$$
\begin{aligned}
a & =-4 d_{\mathrm{R}}(1-x d)\left(\widetilde{\Omega}_{\mathrm{R}} P+N_{\mathrm{R}}\right)+2 d_{1}\left(\widetilde{\Omega}_{1} P+2 N_{1}\right), \\
b & =4 d_{\mathrm{R}}(1-x d)\left(\widetilde{\Omega}_{\mathrm{R}} P+N_{\mathrm{R}}\right), \\
c & =-d_{\mathrm{R}}(1-x d)\left(\widetilde{\Omega}_{\mathrm{R}} P+N_{\mathrm{R}}\right) .
\end{aligned}
$$

Phương trình (29) chỉ có một nghiệm dương, và nghiệm đó chính là giá trị của $\lambda$ để $f(\lambda)$ đạt cực tiểu. Từ đó, giá trị tối ưu cần tìm là

$$
\lambda^{*}=\frac{-2 d_{\mathrm{R}}(1-x d)\left(\widetilde{\Omega}_{\mathrm{R}} P+N_{\mathrm{R}}\right)+\sqrt{\Delta}}{-4 d_{\mathrm{R}}(1-x d)\left(\widetilde{\Omega}_{\mathrm{R}} P+N_{\mathrm{R}}\right)+2 d_{1}\left(\widetilde{\Omega}_{1} P+2 N_{1}\right)},
$$

trong đó

$$
\Delta=2 d_{1} d_{\mathrm{R}}(1-x d)\left(\widetilde{\Omega}_{\mathrm{R}} P+N_{\mathrm{R}}\right)\left(\widetilde{\Omega}_{1} P+2 N_{1}\right) .
$$

Trong trường hợp lý tưởng, hệ số phân bổ công suất tối ưu được xác định bởi

$$
\lambda^{* \mathrm{id}}=\frac{-2\left(\widetilde{\Omega}_{\mathrm{R}} P+N_{\mathrm{R}}\right)+\sqrt{\Delta^{\mathrm{id}}}}{-4\left(\widetilde{\Omega}_{\mathrm{R}} P+N_{\mathrm{R}}\right)+2\left(\widetilde{\Omega}_{1} P+2 N_{1}\right)},
$$

trong đó

$$
\Delta^{\mathrm{id}}=2\left(\widetilde{\Omega}_{\mathrm{R}} P+N_{\mathrm{R}}\right)\left(\widetilde{\Omega}_{1} P+2 N_{1}\right) .
$$

\section{KẾT QUẢ TÍNH TOÁN SỐ VÀ THẢO LUÂN}

Để kiểm chứng kết quả phân tích, tính toán $\mathrm{OP}, \mathrm{SEP}$ và phân bổ công suất tối ưu cho hệ thống khảo sát, chúng tôi sử dụng mô phỏng Monte Carlo, xem xét ảnh hưởng của lỗi phần cứng và mức độ nhiễu dư đến phẩm chất hệ thống. Trong đó tỉ số SNR trung bình được xác định bằng biểu thức $\mathrm{SNR}=P_{0} / N_{0}$, với $P_{0}=P / 3$ và $N_{0}=N_{1}=N_{2}=N_{\mathrm{R}}=1$.
Hiệu năng hệ thống được tập trung khảo sát tại $S_{2}$, kết quả tại $\mathrm{S}_{1}$ được xác định bằng phương pháp tương tự. Các tham số còn lại sử dụng trong mô phỏng và đánh giá như sau: $\Omega_{1}=\Omega_{2}=1$ và $\widetilde{\Omega}_{1}=\widetilde{\Omega}_{2}=\widetilde{\Omega}_{R}=\widetilde{\Omega}$.

Hình 2 minh họa xác suất dừng tại $\mathrm{S}_{2}$ theo $\mathrm{SNR}$ trung bình, trong đó chất lượng hệ thống với phần cứng không lý tưởng được so sánh với hệ thống có phần cứng lý tưởng (ideal hardware). Đồng thời, phẩm chất $\mathrm{OP}$ khi sử dụng phân bổ công suất tối ưu $\left(\lambda=\lambda^{*}\right)$ ở các biểu thức (30) và (31) được so sánh với phân bổ công suất ngẫu nhiên $(\lambda=1 / 10)$, với mức ngưỡng khảo sát cho OP là $x=1$.

Trên cơ sở những kết quả phân tích, đo lường và thử nghiệm về lỗi phần cứng tích hợp cũng như khả năng SIC cho hệ thống FD, chúng tôi khảo sát phẩm chất hệ thống với lỗi phần cứng và $\mathrm{RSI}$ tương ứng là $k_{1}=k_{2}=k_{\mathrm{R}}=k=0,1$ và $\widetilde{\Omega}=-30 \mathrm{~dB}$. Đường lý thuyết chính xác và xấp xỉ thể hiện kết quả ở các biểu thức (17) và (21) cho trường hợp phần cứng không lý tưởng, và biểu thức (19) cho trường hợp phần cứng lý tưởng.

Mức độ ảnh hưởng của lỗi phần cứng đến $\mathrm{OP}$ hệ thống là lớn, đặc biệt ở vùng SNR cao (trên $30 \mathrm{~dB}$ ). Lỗi phần cứng kết hợp với nhiễu dư, làm cho $\mathrm{OP}$ sớm đạt mức bão hòa. Điều này thể hiện rõ trong kết quả tính toán xác suất dừng, bằng cách cho công suất phát tiến đến vô hạn, ta sẽ thu được biểu thức $\mathrm{OP}$ là một hằng số khác 0 . Trong khi đó, với phần cứng lý tưởng, OP tiếp tục giảm khi tăng SNR. Mặt khác, phân bổ công suất tối ưu cho độ lợi đáng kể so với trường hợp ngẫu nhiên, đặc biệt cho hệ thống lý tưởng. Chú ý rằng, với những tham số đã chọn, phân bổ công suất tối ưu có giá trị từ 0,25 đến 0,3 cho cả hệ thống lý tưởng và không lý tưởng.

Hình 3 thể hiện xác suất dừng tại $\mathrm{S}_{2}$ theo hệ số phân bổ công suất, với mức nhiễu dư và lỗi phần cứng khác nhau.

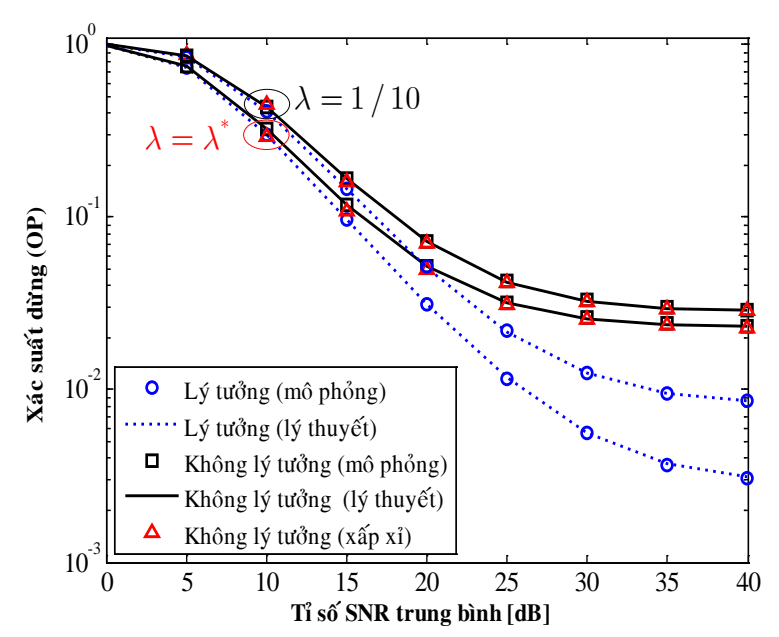

Hình 2. Ảnh hưởng của lỗi phần cứng và hệ số phân bổ công suất đến xác suất dừng tại $\mathrm{S}_{2}$. 
Trong đó, xác suất dừng được khảo sát với $\mathrm{SNR}=40 \mathrm{~dB}$ cho hai trường hợp: (i) $k=0,05$ và $\widetilde{\Omega}=-25 \mathrm{~dB}$, (ii) $k=0,1$ và $\widetilde{\Omega}=-30 \mathrm{~dB}$, các tham số còn lại sử dụng như ở Hình 2.

Trong trường hợp (i), phẩm chất $\mathrm{OP}$ cho hệ thống lý tưởng và không lý tưởng có sự thay đổi rõ rệt theo $\lambda$. Khi ta tăng mức độ lỗi phần cứng, từ 0,05 ở trường hợp (i) lên 0,1 ở trường hợp (ii), OP hệ thống không lý tưởng đạt tới bão hòa, do đó không có sự khác biệt khi $\lambda$ thay đổi. Kết quả cho thấy sự đúng đắn của kết quả phân tích so với kêt quả khảo sát. Đồng thời khi lỗi phần cứng lớn, kết hợp với công suất phát đủ lớn, có thể sử dụng phân bổ công suất cố định cho hệ thống để giảm độ phức tạp.

Hình 4 khảo sát $\mathrm{OP}$ tại $\mathrm{S}_{2}$ theo mức độ ảnh hưởng của lỗi phần cứng, với $\mathrm{SNR}=40 \mathrm{~dB}$ và $\widetilde{\Omega}=-30 \mathrm{~dB}$, sử dụng phân bổ công suất tối ưu và tốc độ truyền dẫn thay đổi, $\mathcal{R}=1 ; 2 ; 3 \mathrm{bit} / \mathrm{s} / \mathrm{Hz}$. Chú ý rằng khi $k_{1}=k_{2}=k_{\mathrm{R}}=k=0$, phẩm chất $\mathrm{OP}$ của hệ thống khảo sát trở thành $\mathrm{OP}$ cho hệ thống lý tưởng. Kết quả cho thấy sự ảnh hưởng lớn của lỗi phần cứng đến phẩm chất hệ thống. Ngay cả khi lỗi rất nhỏ $(k=0,01)$, phẩm chất hệ thống bắt đầu giảm so với hệ thống lý tưởng. Khi lỗi lớn $(k=0,15)$ phẩm chất hệ thống giảm 10 lần so với hệ thống lý tưởng.

Hình 5 minh họa tổng quan về xác suất dừng dưới sự ảnh hưởng đồng thời của hai yếu tố là lỗi phần cứng và khả năng triệt nhiễu tự giao thoa với $\mathrm{SNR}=40 \mathrm{~dB}$ và $\lambda=0,3$. Trong trường hợp $k=0$ và $\widetilde{\Omega}=0$, hệ thống khảo sát trở thành hệ thống $\mathrm{HD}$ với phần cứng lý tưởng. Cả lỗi phần cứng và nhiễu tự giao thoa đều ảnh hưởng lớn đến phẩm chất hệ thống, vì vậy để hệ thống FD hai chiều có thể hoạt động được, các giải pháp kỹ thuật về triệt nhiễu tự giao thoa và thiết kế phần cứng cần được tiến hành đồng bộ và có hiệu quả.

Hình 6 so sánh thông lượng hệ thống xem xét (Throughput - bit/s/Hz) với hệ thống lý tưởng với $k=0,1$; $\widetilde{\Omega}=-30 \mathrm{~dB}$ và $\lambda=\lambda^{*}$. Trong đó, thông lượng hệ thống được xác định thông qua biểu thức $\mathcal{T}=\mathcal{R}(1-\mathrm{OP})$. Với tốc độ truyền dẫn thấp $(\mathcal{R}=2)$, ảnh hưởng của lỗi phần cứng đến thông lượng không đáng kể. Nhưng khi tốc độ truyền dẫn tăng lên $(\mathcal{R}=3 ; 4)$, hệ thống với lỗi phần cứng không thể đạt tới tiệm cần yêu cầu ngay cả khi SNR đủ lớn. Khi $\mathcal{R}=4 \mathrm{bit} / \mathrm{s} / \mathrm{Hz}$, hệ thống không lý tưởng chỉ đạt $\mathcal{T}=2,5$ bit/s/Hz. Vì vậy, cho hệ thống FD truyền dẫn tốc độ cao, việc sử dụng các thiết bị phần cứng giá thành cao để đảm bảo phẩm chất hệ thống là điều tất yếu.

Hình 7 minh họa phẩm chất lỗi ký hiệu tại $\mathrm{S}_{2}$ theo $\mathrm{SNR}$ trung bình, sử dụng điều chế BPSK với $k=0,1 ; \widetilde{\Omega}=$ $-30 \mathrm{~dB}$ và $\lambda=1 / 3$ (tức là $P_{1}=P_{2}=P_{\mathrm{R}}$ ). Trong đó, đường lý thuyết thể hiện kết quả ở biểu thức (25) và (26). Ta thấy rằng, khi SNR thấp (dưới $10 \mathrm{~dB}$ ) sự sai khác giữa phân tích lý thuyết và kết quả mô phỏng là khá lớn. Nhưng

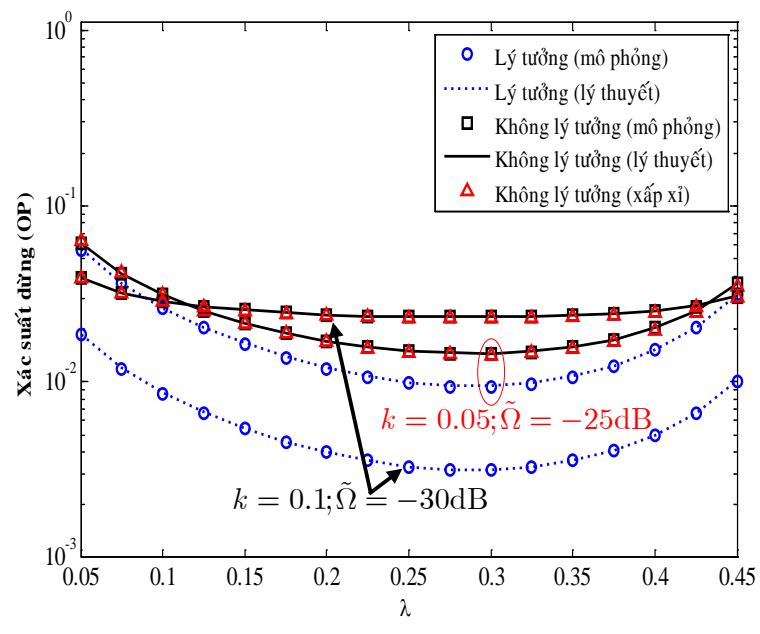

Hình 3. Xác suất dừng tại $\mathrm{S}_{2}$.

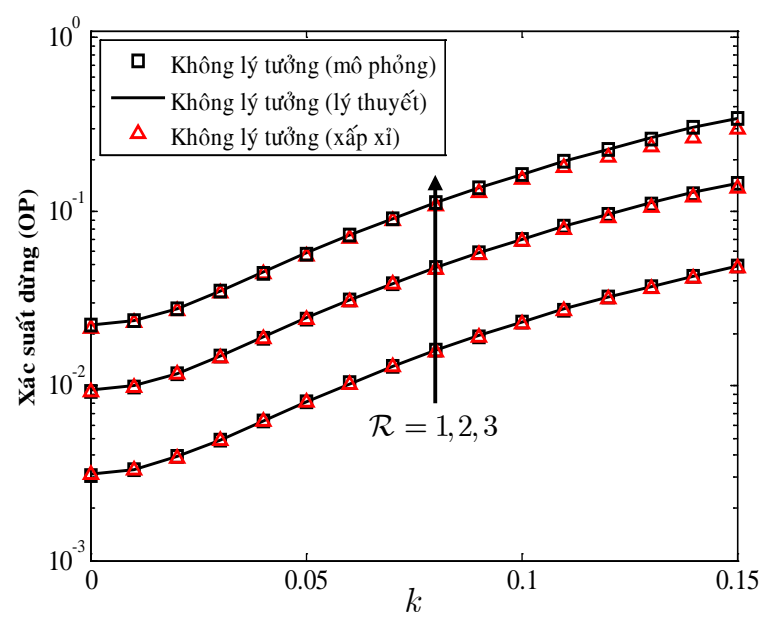

Hình 4. Ảnh hưởng của lỗi phần cứng $k$ tới xác suất dừng tại $\mathrm{S}_{2}$ với tốc độ truyền dẫn khác nhau.

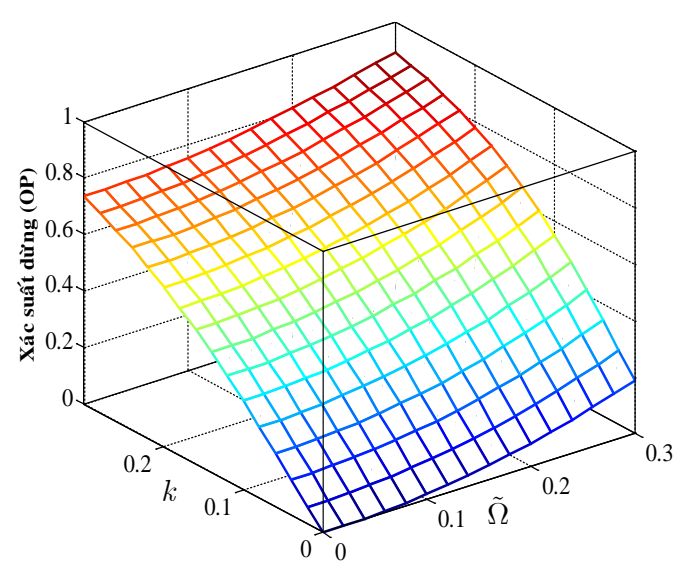

Hình 5. Xác suất dừng tại $\mathrm{S}_{2}$ theo lỗi phần cứng $k$ và khả năng triệt nhiễu $\widetilde{\Omega}$. 


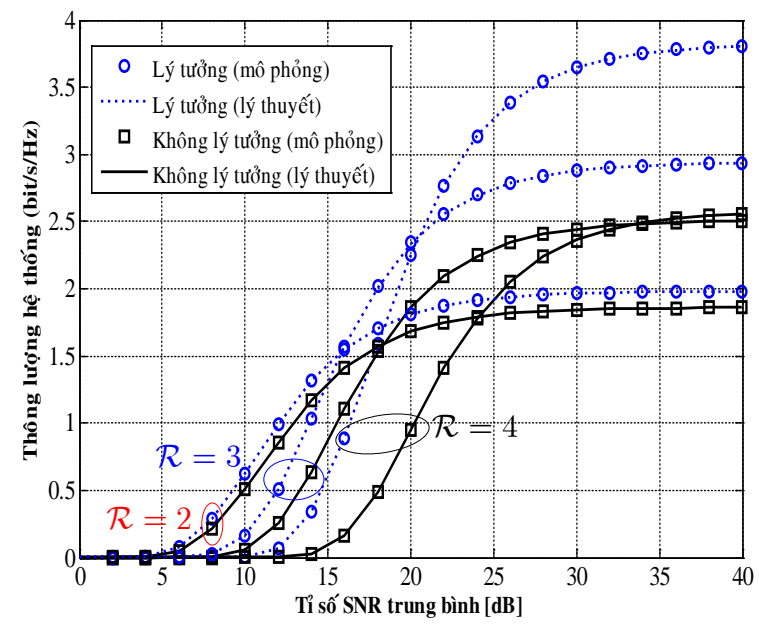

Hình 6. Thông lượng hệ thống theo sự thay đổi SNR và tốc độ truyền dẫn khác nhau.

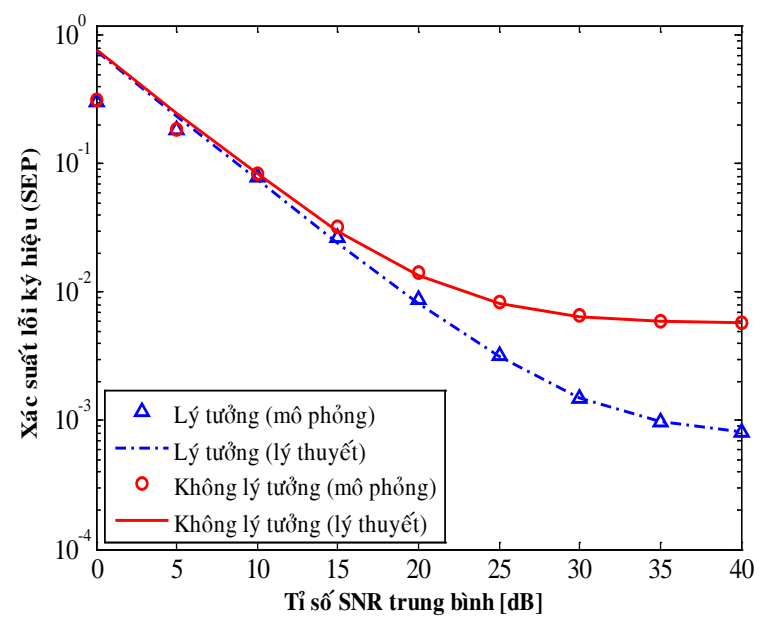

Hình 7. Xác suất lỗi ký hiệu tại $\mathrm{S}_{2}$ theo $\mathrm{SNR}$, với $P_{1}=P_{2}=P_{\mathrm{R}}$.

ở vùng $\mathrm{SNR}$ cao (trên $10 \mathrm{~dB}$ ) kết quả tính toán hoàn toàn đúng với kết quả mô phỏng. Đồng thời, với $\mathrm{SNR}=40 \mathrm{~dB}$, hệ thống với lỗi phần cứng có SEP cao hơn 10 lần so với hệ thống lý tưởng.

\section{KẾT LUÂ̂N}

Bằng phương pháp phân tích giải tích, biểu thức chính xác và gần đúng của xác suất dừng và xác suất lỗi ký hiệu đã được đưa ra cho hệ thống truyền dẫn song công hai chiều trong trường hợp có lỗi phần cứng. Đồng thời, để nâng cao phẩm chất hệ thống, chúng tôi đưa ra giá trị phân bổ công suất tối ưu cho hai nút đầu cuối và nút chuyển tiếp. Kết quả phân tích đã chứng minh sự ảnh hưởng của lỗi phần cứng và nhiễu tự giao thoa còn sót lại đến phẩm chất hệ thống khi so sánh với hệ thống lý tưởng, đặc biệt cho hệ thống truyền dẫn tốc độ cao. So với hệ thống lý tưởng, hệ thống có phần cứng không lý tưởng có phẩm chất kém hơn nhiều lần, nhất là trong trường hợp hệ số méo dạng lớn. Bên cạnh đó, so với hệ thống HD lý tưởng thì hệ thống FD không lý tưởng có phẩm chất kém hơn nhiều và sớm đạt tới mức bão hòa khi công suất phát đủ lớn. Vì vậy, ngoài việc thực hiện tốt các kỹ thuật để triệt nhiễu tự giao thoa cho truyền thông song công trên cùng băng tần, các nhà sản xuất cần thực hiện tốt việc hạn chế lỗi phần cứng nhằm làm giảm tác động tới phẩm chất hệ thống, từ đó tạo tính khả thi cho truyền thông song công trên cùng băng tần.

\section{TÀI LIỆU THAM KHẢO}

[1] D. Choi and J. H. Lee, "Outage probability of two-way fullduplex relaying with imperfect channel state information," IEEE Communications Letters, vol. 18, no. 6, pp. 933-936, June 2014.

[2] H. Cui, M. Ma, L. Song, and B. Jiao, "Relay selection for two-way full duplex relay networks with amplify-andforward protocol," IEEE Transactions on Wireless Coтmunications, vol. 13, no. 7, pp. 3768-3777, Jul. 2014.

[3] A. Sabharwal, P. Schniter, D. Guo, D. W. Bliss, S. Rangarajan, and R. Wichman, "In-band full-duplex wireless: Challenges and opportunities," IEEE Journal on Selected Areas in Communications, vol. 32, no. 9, pp. 1637-1652, 2014.

[4] D. Bharadia, E. McMilin, and S. Katti, "Full duplex radios," in Proceedings of the ACM SIGCOMM Conference (SIGCOMM '13). New York, USA: ACM, 2013, pp. 375-386.

[5] Y. Y. Kang, B.-J. Kwak, and J. H. Cho, "An optimal fullduplex af relay for joint analog and digital domain selfinterference cancellation," IEEE Transactions on Communications, vol. 62, no. 8, pp. 2758-2772, 2014.

[6] D. Korpi, L. Anttila, V. Syrjälä, and M. Valkama, "Widely-linear digital self-interference cancellation in direct-conversion full-duplex transceiver," arXiv preprint arXiv:1402.6083, 2014.

[7] J. R. Krier and I. F. Akyildiz, "Active self-interference cancellation of passband signals using gradient descent," in Proceedings of the IEEE 24th International Symposium on Personal Indoor and Mobile Radio Communications (PIMRC). IEEE, 2013, pp. 1212-1216.

[8] E. Ahmed and A. M. Eltawil, "All-digital self-interference cancellation technique for full-duplex systems," IEEE Transactions on Wireless Communications, vol. 14, no. 7, pp. 3519-3532, 2015.

[9] T. M. Kim and A. Paulraj, "Outage probability of amplifyand-forward cooperation with full duplex relay," in Proceedings of the Wireless Communications and Networking Conference (WCNC). IEEE, Apr. 2012, pp. 75-79.

[10] C. H. de Lima, H. Alves, P. H. Nardelli, and M. Latvaaho, "Effects of relay selection strategies on the spectral efficiency of wireless systems with half-and full-duplex nodes," IEEE Transactions on Vehicular Technology, vol. 66, no. 8, pp. 7578-7583, 2017.

[11] T. Riihonen, S. Werner, and R. Wichman, "Hybrid fullduplex/half-duplex relaying with transmit power adaptation," IEEE Transactions on Wireless Communications, vol. 10, no. 9, pp. 3074-3085, Sept. 2011.

[12] J. Hu, F. Liu, and Y. Liu, "Achievable rate analysis of twoway full duplex relay with joint relay and antenna selection," in Proceedings of the IEEE Wireless Communications and Networking Conference (WCNC), March 2017, pp. 1-5.

[13] A. Koc, I. Altunbas, and A. Yongaçoglu, "Outage probability of two-way full-duplex af relay systems over nakagami-m 
fading channels," in Proceedings of the IEEE 84th Vehicular Technology Conference (VTC-Fall). IEEE, 2016, pp. 1-5.

[14] X. Li, C. Tepedelenlioglu, and H. Senol, "Channel estimation for residual self-interference in full duplex amplify-andforward two-way relays," IEEE Transactions on Wireless Communications, vol. 16, no. 8, pp. 4970-4983, 2017.

[15] Y. Li, N. Li, M. Peng, and W. Wang, "Relay power control for two-way full-duplex amplify-and-forward relay networks," IEEE Signal Processing Letters, vol. 23, no. 2, pp. 292-296, 2016.

[16] Y. Wang, Q. Jiang, Z. Chen, and B. Xia, "Outage probability of two-way full-duplex amplify-forward relay systems with asymmetric traffic requirements," in Proceedings of the International Conference on Wireless Communications \& Signal Processing (WCSP). IEEE, 2015, pp. 1-5.

[17] G. Chen, P. Xiao, J. R. Kelly, B. Li, and R. Tafazolli, "Full-duplex wireless-powered relay in two way cooperative networks," IEEE Access, vol. 5, pp. 1548-1558, 2017.

[18] E. Fidan and O. Kucur, "Performance of transceiver antenna selection in two way full-duplex relay networks over rayleigh fading channels," IEEE Transactions on Vehicular Technology, 2018.

[19] A. Hyadi, M. Benjillali, and M. S. Alouini, "Outage performance of decode-and-forward in two-way relaying with outdated CSI," IEEE Transactions on Vehicular Technology, vol. 64, no. 12, pp. 5940-5947, 2015.

[20] C. Li, Z. Chen, Y. Wang, Y. Yao, and B. Xia, "Outage analysis of the full-duplex decode-and-forward two-way relay system," IEEE Transactions on Vehicular Technology, vol. 66, no. 5, pp. 4073-4086, May 2017.

[21] C. Li, H. Wang, Y. Yao, Z. Chen, X. Li, and S. Zhang, "Outage performance of the full-duplex two-way DF relay system under imperfect CSI," IEEE Access, vol. 5, pp. 54255435, 2017.

[22] C. Li, B. Xia, S. Shao, Z. Chen, and Y. Tang, "Multiuser scheduling of the full-duplex enabled two-way relay systems," IEEE Transactions on Wireless Communications, vol. 16, no. 2, pp. 1094-1106, Feb. 2017.

[23] B. Zhong and Z. Zhang, "Secure full-duplex two-way relaying networks with optimal relay selection," IEEE Communications Letters, vol. 21, no. 5, pp. 1123-1126, May 2017.

[24] E. Bjornson, M. Matthaiou, and M. Debbah, "A new look at dual-hop relaying: Performance limits with hardware impairments," IEEE Transactions on Communications, vol. 61, no. 11, pp. 4512-4525, Nov. 2013.

[25] A. Papazafeiropoulos, S. Sharma, T. Ratnarajah, and S. Chatzinotas, "Impact of residual additive transceiver hardware impairments on rayleighproduct mimo channels with linear receivers: Exact and asymptotic analyses," IEEE Transactions on Communications, 2018.

[26] O. Taghizadeh, A. C. Cirik, and R. Mathar, "Hardware impairments aware transceiver design for full-duplex amplifyand-forward mimo relaying," IEEE Transactions on Wireless Communications, vol. 17, no. 3, pp. 1644-1659, 2018.

[27] Y. Liu, X. Xue, J. Zhang, X. Li, L. Dai, and S. Jin, "Multipair massive mimo two-way full-duplex relay systems with hardware impairments," in Proceedings of the IEEE Global Communications Conference (GLOBECOM). IEEE, 2017, pp. 1-6.

[28] A. Jeffrey and D. Zwillinger, Table of integrals, series, and products. Academic press, 2007.

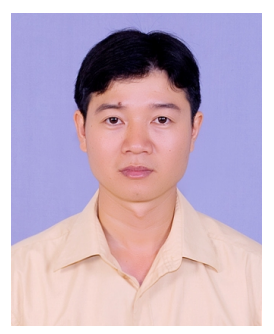

Nguyễn Bá Cao tốt nghiệp Trường Sĩ quan Thông tin năm 2006, nhận bằng Thạc sĩ tại Học viện Công nghệ Bưu chính Viễn thông năm 2011. Hiện nay, tác giả đang là nghiên cứu sinh năm thứ hai tại Khoa Vô tuyến điện tử, Học viện Kỹ thuật Quân sự. Lĩnh vực nghiên cứu của tác giả là truyền thông song công, truyền thông hợp tác.

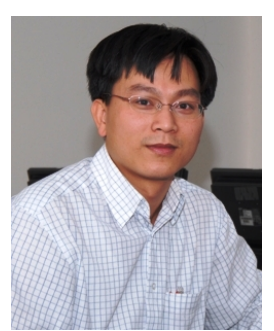

Trần Xuân Nam nhận bằng Thạc sĩ tại Đại học Công nghệ, Sydney, Úc, năm 1998; nhận bằng Tiến sĩ tại Đại học Điện tử Truyền thông, Tokyo, Nhật Bản, năm 2006. Hiện nay, tác giả đang là phó giáo sư và công tác tại Học viện Kỹ thuật Quân sự. Tác giả đã được tặng giải thưởng nhà nghiên cứu trẻ tại Nhật Bản năm 2003 và giải thưởng bài báo xuất sắc nhất tại hội nghị ATC năm 2012. Tác giả là thành viên của IEEE, IEICE và Hiệp hội Vô tuyến Điện tử Việt Nam. Lĩnh vực nghiên cứu của tác giả là ăng-ten thích ứng, xử lý tín hiệu, mã hóa và hệ thống MIMO. 